\title{
Grenzüberschreitende Effekte von Kapazitätsmechanismen
}

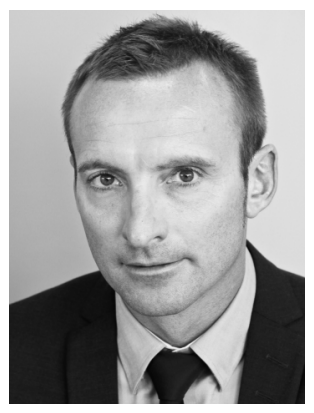

\section{Urs Meister}

Energie, Investitionsentscheidung, Kapazitätsmarkt, Externe Effekte, Energy-only-Markt, Internationaler Handel

Energy, Investment Decision, Capacity Market, Externalities, Energy-only-Market, International Trade

Mit der Energiewende und dem wachsenden Anteil Erneuerbarer nehmen die Zweifel an der Stabilität eines ,Energy-Only' Elektrizitätsmarktdesigns zu. Einige europäische Länder erwägen daher die Einführung von Kapazitätsmechanismen, die eine separate Abgeltung für die Bereitstellung von Back-up-Kraftwerken vorsehen und Investitionsanreize für konventionelle Kraftwerke schaffen. Die wissenschaftliche Literatur gibt eine Übersicht über die Ausgestaltung sowie Vor- und Nachteile von Kapazitätsmechanismen. Wenig Beachtung finden dagegen die damit verbundene Externalitäten zwischen benachbarten Strommärkten. Der folgende Beitrag analysiert die Auswirkungen national definierter Kapazitätsmechanismen im eng vernetzten europäischen Markt. Er illustriert, dass vor allem kleine, handelsorientierte Länder von den Externalitäten betroffen sind. Bei der Wahl ihres Strommarktdesigns sind sie faktisch dazu gezwungen, den Regulierungsrahmen grösserer Nachbarländer zu imitieren, um negative Auswirkungen etwa auf die eigene Versorgungssicherheit zu verhindern.

The energy transition policy and the increasing share of renewables put the sustainability of the Energy-only-Market design in question. Some European countries intend to change such market design by introducing an additional capacity mechanism in order to support the provision of backup power plants separately. Such measure would stimulate incentives to invest into conventional power stations. Previous scientific literature offers a broad overview of the design options, benefits and drawbacks of these mechanisms. However, there is a lack of research regarding the induced externalities between neighbouring electricity markets. This article considers the strongly interconnected European market and analyses the implications of country-specific capacity mechanisms. It shows that small countries with a high volume of cross border trade are most badly affected by externalities. In order to avoid negative impacts (e.g. regarding security of supply), they need to imitate their neighbours' regulatory framework.

\section{Einführung}

In vielen europäischen Ländern wachsen die Zweifel an einer längerfristigen Vereinbarkeit von Energiewende und dem Energy-only-Markt, auf dem lediglich Energie als Megawattstunden $(\mathrm{MWh})$ gehandelt wird. Die steigende (subventionierte) Einspeisung erneuerbarer Energien lässt die Energiepreise im Markt sinken, immer häufiger sogar gegen null konver- 
gieren. Nach Ansicht vieler Branchenvertreter stellt dies die Wirtschaftlichkeit von konventionellen Kraftwerken zunehmend in Frage. Vermehrt wird daher die Einführung sogenannter Kapazitätsmechanismen gefordert, um Anreize für den Bau konventioneller Kraftwerke zu schaffen. Denn diese sind als Back-up dringend nötig, um die fluktuierende Produktion erneuerbarer Energien auszugleichen. Kapazitätsmechanismen sind keine neue Erscheinung. Schon zu Beginn der Strommarktliberalisierungen in den 1990er Jahren - also noch bevor der Begriff der Energiewende bekannt war - wurde die Stabilität des Energyonly-Marktes angezweifelt. Ungewissheit besteht darüber, ob der Markt ausreichend Investitionsanreize für jene (Spitzenlast-) Kraftwerke gibt, die relativ selten zum Einsatz kommen, aber für die Systemstabilität nötig sind. Aus ökonomischer Sicht geht es dabei um die vermutete Existenz eines Marktversagens. Viele Diskurse drehen sich daher um die Fragen der Notwendigkeit sowie der Ausgestaltung von Kapazitätsmechanismen.

Kaum thematisiert werden durch Kapazitätsmechanismen implizierte ,Externalitäten zwischen benachbarten Strommärkten. Zwar können die Mechanismen national eingeführt, betrieben und finanziert werden, doch entfaltet sich ihre Wirkung über die Preiseffekte im Energiemarkt auch auf benachbarte Märkte. Solche externen Effekte sind dann vernachlässigbar, wenn der grenzüberschreitende Handel im Vergleich zum inländischen Markt verschwindend klein ist. Kleine, offene Strommärkte - wie beispielsweise die Schweiz oder Österreich - mit relativ hohem Handelsanteil sind dagegen in hohem Ausmass von solchen Effekten betroffen, da ihre Stromgrosshandelspreise wesentlich durch die marktlichen Gegebenheiten in den Nachbarländern bestimmt werden. Dadurch reduziert sich die Effektivität eines eigenen Kapazitätsmechanismus. Umgekehrt, wenn ein kleiner offener Strommarkt selber keinen Kapazitätsmechanismus installiert, werden die Wirkungen von ausländischen Kapazitätsmechanismen über den Energiehandel importiert. Konsequenterweise können kleine Märkte keine autonome Strategie hinsichtlich der Kapazitätsmechanismen wählen - vielmehr braucht es eine gewisse Koordination mit den Entwicklungen in den Nachbarländern. Für die Kraftwerksbetreiber und -investoren in kleinen offenen Strommärkten resultieren dadurch zusätzliche (regulatorische) Risiken.

\section{Preise und Investitionsanreize}

In wettbewerblichen Märkten gilt der Grundsatz, dass der Preismechanismus alleine fähig ist, die richtigen Investitionssignale zu geben. Dass im Strommarkt gewisse Zweifel bestehen, hat mit der Relevanz und Fragilität der Versorgungssicherheit zu tun. Einerseits sind die Kosten eines Black-outs ausserordentlich hoch. So werden etwa die gesamtwirtschaftlichen Kosten eines eintägigen Stromausfalls in Deutschland auf 14 bis 30 Mrd. Euro geschätzt (Bothe/Riechmann 2008). Anderseits stellt die Versorgungsstabilität im liberalisierten Strommarkt eine Art öffentliches Gut dar: Im Falle eines Kraftwerkausfalls und einer damit verbundenen Systeminstabilität ist nicht nur der vertragliche Abnehmer betroffen, sondern die Versorgung als Ganzes. Ein einzelner Akteur würde bei seinen Entscheidungen die immensen Kosten eines solchen Systemausfalls zu wenig berücksichtigen. Aus diesem Grund hat der Betreiber des Übertragungsnetzes die Funktion eines Systemoperators: Er stellt im Falle kurzfristiger Ungleichgewichte - beispielsweise bei Prognoseabweichungen oder Kraftwerksausfällen - die nötigen Reserven bereit. Solche Systemdienstleistungen sind jedoch kein Instrument zur Schaffung von Investitionsanreizen, die eine langfristige Versorgungssicherheit im Sinne ausreichender Produktionskapazitäten garantieren. Diese Funktion kommt im liberalisierten Markt alleine dem Preismechanismus zu. 
Doch besteht eine gewisse Unsicherheit darüber, ob der Markt fähig ist, ausreichend Investitionsanreize für jene Kraftwerke zu schaffen, die relativ wenig zum Einsatz kommen, aber für die Stabilität in Extremsituationen wichtig sind. Denn in einem wettbewerblichen Markt bildet sich die Angebotskurve auf Basis der kurzfristigen Grenzkosten. Das heisst, die Abgeltung des letzten noch eingesetzten Kraftwerks basiert auf seinen Grenzkosten. Spitzenlastkraftwerke weisen daher nicht nur wenige Vollbenutzungsstunden auf. Gleichzeitig profitieren sie auch nicht oder nur selten von Preisen, die über ihren Grenzkosten liegen - jedenfalls wenn hinreichend Kraftwerke zur Verfügung stehen und Wettbewerb herrscht (Cramton/Stoft 2006). In der Literatur wird dieser Umstand als Missing Money Problem bezeichnet - es fehlt an Mitteln zur Deckung der Fixkosten. Aus ökonomischer Sicht ist der Begriff jedoch strittig. Schliesslich funktionieren auch andere Märkte nach demselben Muster: Angebot und Nachfrage bestimmen den Preis, die Angebotskurve ihrerseits wird durch die Grenzkosten gebildet. Investitionsanreize entstehen dabei auf zwei Arten: Erstens können neue Technologien höhere Wirkungsgrade erzielen und profitieren, wenn ältere Technologien am Markt die Preise bestimmen. Zweitens schlägt sich ein mangelndes Kraftwerksangebot in besonders hohen - kurzzeitig auftretenden - Knappheitspreisen nieder. Solche Preise könnten sich theoretisch an den hohen Kosten eines Versorgungsausfalls orientieren, dem Value of Lost Load (VoLL). Häufig beläuft sich der geschätzte Wert des VoLL auf das 10- bis 100-fache der durchschnittlichen Strommarktpreise (Frontier Economics 2008), einige vermuten sogar Werte bis zum 500-fachen (Joskow/ Tirole 2007). Derart hohe Preise wären - auch wenn sie sich nur kurzzeitig einstellen grundsätzlich fähig, ausreichende Investitionsanreize vor allem für Anlagen mit tiefen Fixkosten und hohen marginalen Kosten zu geben. Doch besteht die Gefahr, dass ein Regulator solche Preisspitzen nicht zulassen würde - etwa weil er dahinter Marktmacht oder Absprachen vermuten würde. Ausserdem könnten auch Preise, die unter dem VoLL-Niveau liegen, noch ausreichende Investitionsanreize schaffen (Meister 2013).

Das Missing Money Problem ist als solches nicht neu. Zweifellos aber hat es sich mit der Energiewende akzentuiert. Da erneuerbare Energien wie Wind oder Photovoltaik (PV) praktisch keine marginalen Kosten aufweisen, schiebt sie die Angebotskurve (Merit Order) im Markt parallel nach rechts. Damit reduziert das zusätzliche (subventionierte) Angebot die durchschnittlichen Preise am Markt. Gleichzeitig nimmt die Auslastung konventioneller Kraftwerke rechts in der Merit Order (also vor allem der Spitzenlastkraftwerke) weiter ab. Dies führt zu einer weiteren Schwächung der Investitionsanreize bei den konventionellen, nicht-subventionierten Kraftwerken, die aber dringend als Back-up für Perioden mit wenig Wind oder Sonne nötig sind.

\section{Gestaltung und Wirkung von Kapazitätsmechanismen}

Eine Möglichkeit zur Adressierung des Missing-Money-Problems ist die Einführung sogenannter Kapazitätsmechanismen. Sie sind komplementär zum Energiemarkt (auf dem Energie als MWh gehandelt wird) und ermöglichen eine Abgeltung der blossen Vorhaltung von Kraftwerkskapazitäten (MW). Solche Mechanismen sind keineswegs neu, vielmehr existieren sie in der einen oder anderen Form schon seit den Jahren. Häufig wird zwischen preis- und mengenbasierten Mechanismen unterschieden (Tietjen 2012; Barrera et al. 2011; Siegmeier 2011). Im Falle von preisbasierten Mechanismen entscheidet eine zentrale Instanz über eine finanzielle Abgeltung (administrative Kapazitätszahlungen, strategische Reserve), bei mengenbasierten Instrumenten entscheidet sie über die Kapazitäten, 
während sich der Preis dafür in einem Markt bildet (Kapazitätsverpflichtungen). Häufig werden lediglich mengenbasierte Instrumente als Kapazitätsmärkte im engeren Sinn bezeichnet.

Die sogenannte administrative Kapazitätszablung gilt als einfache Form eines preisbasierten Mechanismus. Solche Zahlungen - wie sie etwa in Spanien existieren - werden den Kraftwerksbetreibern als Ergänzung zu den Erträgen aus dem Energiemarkt ausgerichtet. Die Zuschüsse werden administrativ festgelegt und meist etwa so berechnet, dass (Spitzenlast-) Kraftwerke ihre ungedeckten Fixkosten finanzieren können. Die Zahlung kann als jährlicher, fixer Betrag festgelegt werden oder er wird nach Verfügbarkeit oder gar effektiver Produktion während bestimmter Zeitperioden differenziert (Pfeifenberger et al 2009) gerade bei den produktionsbezogenen Zahlungen resultieren allerdings Marktverzerrungen, ähnlich wie bei der Förderung Erneuerbarer via Einspeisevergütung. Die strategische Reserve stellt einen alternativen preisbasierten Mechanismus dar. Hier beschafft der Systemoperator zusätzliche Kraftwerkskapazitäten, etwa im Rahmen eines Auktionsverfahrens, und gibt diese bei Knappheit bzw. einem bestimmten Preisniveau zur Entlastung in den Markt. Der definierte Auslösungspreis wird dadurch zur faktischen Preisobergrenze. Der Auslösungspreis bestimmt zudem, wie gross die Reserve längerfristig sein muss (vgl. auch EWI 2012). Als mengen- und am ehesten marktbasiertes Instrument gilt die Kapazitätsverpflichtung. Sie wird vor allem in den USA angewendet. Dabei werden Versorger (oder Grossverbraucher) angehalten, Kraftwerkskapazitäten basierend auf ihren Bedarfsspitzen vorzuhalten (vgl. Cramton/Stoft 2006; Cramton/Stoft 2008; Bidwell 2005). Dazu können sie a) eigene Kraftwerke betreiben, b) Verträge mit Dritten abschliessen oder c) über einen zentral organisierten Markt entsprechende Kraftwerkszertifikate einkaufen. Auf einem derartigen zentralen Forward-Markt können grundsätzlich auch Verbraucher teilnehmen, die alternativ Lastreduktionsmassnahmen anbieten. Sinnvollerweise werden sowohl Produktionskapazitäten als auch verbrauchsseitige Massnahmen aufgrund ihres tatsächlichen Beitrags zur Systemstabilität gewichtet, also nach ihrer technischen Verfügbarkeit während potenzieller Knappheitsperioden.

Die dargestellten Mechanismen weisen hinsichtlich ihrer Wirkung Parallelen auf. Kapazitätsmechanismen stellen kein grundsätzliches Re-Design des Elektrizitätsmarktes dar. Vielmehr wird der bestehende Energy-only-Markt um ein Instrument ergänzt. Dieses schafft für Kraftwerksbetreiber eine zusätzliche und weniger volatile Ertragsmöglichkeit. Finanziert werden die Mechanismen über eine Abgabe, die von den Verbrauchern auf dem Energie- oder Netztarif geleistet wird. Umgekehrt profitieren die Verbraucher a) von einer höheren Versorgungssicherheit und b) von der Verhinderung besonders ausgeprägter Preisspitzen, die sich im Falle knapper Kraftwerkskapazitäten im Energy-only-Markt bilden würden. Nicht selten werden Kapazitätsmechanismen durch eine explizite Preisregulierung im Stromgrosshandel flankiert. Damit soll verhindert werden, dass Kraftwerksbetreiber gleichzeitig von den Erträgen aus dem Kapazitätsmechanismus sowie von allfälligen Knappheitspreisen (die womöglich künstlich mittels Marktmacht gefördert werden) profitieren können. Die Kombination der Subventionierung erneuerbarer Energien mit der Einführung eines Kapazitätsmechanismus führt in jedem Fall zu einem tieferen durchschnittlichen Preisniveau im Stromgrosshandel. Dadurch resultieren für konventionelle Kraftwerke am Energiemarkt zwar Ertragseinbussen. Diese aber können durch die zusätzlichen (sichereren) Erträge aus dem Kapazitätsmechanismus kompensiert werden. 


\section{Kritische grenzüberschreitende Effekte}

Die Frage des richtigen Marktdesigns ist besonders komplex, wenn allfällige grenzüberschreitende Effekte berücksichtigt werden müssen. Das gilt vor allem für kleine, besonders handelsorientierte Strommärkte wie die Schweiz, die aufgrund ihrer zentralen Nord-SüdTransitfunktion häufig als eine Art Stromdrehscheibe in Europa bezeichnet wird. Die Bedeutung des Stromaussenhandels illustrieren die grenzüberschreitenden physikalischen Stromflüsse: 2012 standen einer Erzeugung von 68 TWh Stromeinfuhren von 31 TWh und Ausfuhren von 32 TWh gegenüber. Die vertraglichen Werte beliefen sich gar auf 87 bzw. 89 TWh (vgl. BFE 2013). Wegen der hohen Relevanz des Handels werden die inländischen Stromgrosshandelspreise üblicherweise durch die Preise in den Nachbarn bestimmt. Faktisch ist die Schweiz Preisnehmerin, da der eigene Kraftwerkspark durch Technologien mit sehr tiefen oder gar inexistenten Grenzkosten dominiert wird. So lag 2012 der Produktionsanteil der Kernkraft bei 36\%, jener der Wasserkraft bei 59\% (vgl. BFE 2013). Je nach Jahreszeit und Importbedarf pendelt der Schweizer Strompreis zwischen dem höheren italienischen und dem meist tieferen deutschen Niveau. Vor allem im Winter, wenn die Schweiz aufgrund der geringeren Wasserkraftproduktion Strom importieren muss, steigt das Preisniveau auf das höhere italienische (vgl. Abbildung 1).

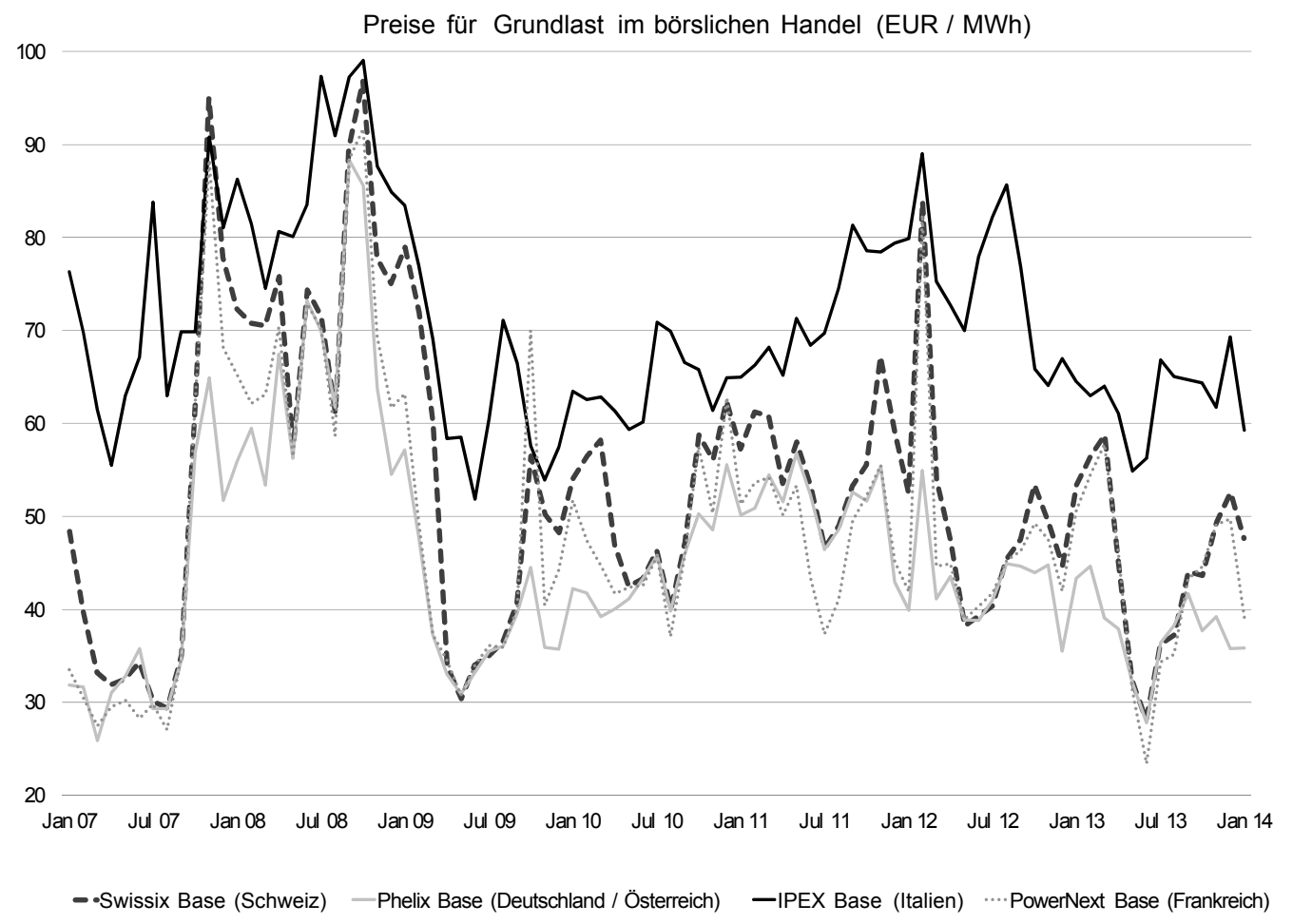

Abb. 1: Preise für Grundlast im börslichen Handel (EUR / MWh) ${ }^{1}$

1 Quelle: Avenir Suisse auf Basis epexspot.com, mercatoelettrico.org. 
Gerade weil der europäische Kontext für ein kleines, handelsorientiertes Land wie die Schweiz derart zentral ist, kann der Effekt eines allfälligen Kapazitätsmechanismus nicht isoliert betrachtet werden. Um die Interaktion mit den benachbarten Märkten zu analysieren, können vereinfachend vier Szenarien unterschieden werden (vgl. Meister 2013): i) Nachbarländer führen Kapazitätsmechanismen ein, die Schweiz dagegen nicht; ii) die Schweiz führt einen Kapazitätsmechanismus ein, die Nachbarn nicht; iii) alle Länder führen Kapazitätsmechanismen ein oder iv) kein Land führt einen solchen Mechanismus ein. Die drei ersten Szenarien werden im Folgenden genauer analysiert.

\section{i) Nachbarländer führen Kapazitätsmechanismen ein, die Schweiz nicht}

In diesem Szenario verzichtet die Schweiz (oder alternativ Österreich) auf einen Kapazitätsmechanismus, während Nachbarländer wie Deutschland, Italien und Frankreich einen solchen Einführen. In diesem Szenario erwirtschaften die konventionellen Kraftwerke im benachbarten Ausland ein Teil ihrer Erträge über einen komplementären Kapazitätsmechanismus (Euro / MW), während ihre Erträge aus dem Energiemarkt (Euro / MWh) tendenziell sinken. Die Kombination zusätzlichen Abgeltungen und Investitionsanreize für konventionelle Anlagen mit einer anhaltenden Subventionierung erneuerbarer Energien führt dazu, dass die durchschnittlichen Preise an den Spot- und Foreward-Märkten sinken. Gleichzeitig nimmt die Wahrscheinlichkeit von kurzzeitig besonders hohen Knappheitspreisen ab. Weil die Schweiz im Grosshandel das tiefere Preisniveau der Nachbarländer übernimmt, können Konsumenten direkt profitieren. Positiv könnten auch die Effekte auf die Versorgungssicherheit sein, sofern die Exporte der Nachbarländer auch während potenziell kritischer Versorgungssituationen wachsen. Dennoch wäre eine reine Trittbrettfahrerstrategie kritisch. Denn die tieferen Preise würden umgekehrt die Erträge der Schweizer Produzenten schmälern und die Investitionsanreize erodieren lassen. Ein Alleingang der Schweiz mit einem Energy-only-Markt könnte daher längerfristig die Versorgungssicherheit unterminieren. Ähnliches gilt im Falle einer Teilnahme der Schweizer Kraftwerksbetreiber an den ausländischen Mechanismen. Schliesslich würden sie dazu verpflichtet, ihre Verfügbarkeit an den Knappheitssituationen im Ausland auszurichten - besonders kritisch wäre das im Zusammenhang mit der (Saison-) Speicherbewirtschaftung.

Aus Sicht der Schweizer Kraftwerksbetreiber stellt sich ausserdem die Frage, ob die Teilnahme an ausländischen Kapazitätsmechanismen die geringeren Erträge aus dem Energiemarkt kompensieren. Doch gerade für die Wasserkraft - deren Anteil in der Schweiz oder auch Österreich besonders hoch ist - dürfte das Potenzial beschränkt sein. Für Pumpspeicherwerke könnte die Rechnung gar negativ sein. Werden Kapazitätsmechanismen marktnahe implementiert, profitieren vor allem Kraftwerkstypen mit tiefen Fixkosten und hoher Verfügbarkeit während den Wintermonaten (in denen eher Knappheitssituationen auftreten). Wasserkraftwerke hingegen weisen hohe Fixkosten auf, zudem produzieren sie vor allem im Sommer. Darüber hinaus reduzieren Kapazitätsmechanismen die Preisvolatilität und stellen damit die Wirtschaftlichkeit von Speichern grundsätzlich in Frage.

\section{ii) Die Schweiz führt einen Kapazitätsmechanismus ein, die Nachbarn nicht}

Möglich ist umgekehrt, dass der kleine, offene Strommarkt im Alleingang einen Kapazitätsmechanismus einführt, während die grossen Nachbarn darauf verzichten. Doch gerade wegen der Vernetzung mit den Nachbarn muss die Effizienz eines solchen Mechanismus 
grundsätzlich in Frage gestellt werden. Im Falle der Schweiz würde ein eigener Kapazitätsmechanismus aufgrund der fremdbestimmten Energiepreise die Entstehung von Knappheitspreisen im Inland nicht verhindern - denn solche würden weiterhin aus dem Ausland importiert. Inländische Verbraucher müssten zwar die Kosten des Mechanismus tragen, könnten aber nicht von einem finanziellen Gegenwert in Form tieferer Grosshandelspreise oder geringerer bzw. seltenerer Preisausschläge (Knappheitspreise) profitieren. Einseitig bevorteilt werden nun die inländischen Kraftwerksbetreiber. Sie profitieren gleichzeitig von den hohen Preisen im Energiemarkt und den Erträgen aus dem Kapazitätsmechanismus. Das Problem könnte theoretisch durch eine Preisregulierung im Grosshandel adressiert werden - schliesslich werden Price Caps häufig im Zusammenhang mit Kapazitätsmechanismen eingeführt. Doch für einen kleinen handelsorientierten Markt wie die Schweiz wäre ein solcher Eingriff besonders verzerrend - gerade weil der Strompreis nicht durch Angebot und Nachfrage auf dem inländischen Markt gebildet wird.

\section{iii) Alle Länder führen einen Kapazitätsmechanismus ein}

Die beiden obigen Szenarien illustrieren, dass ein Alleingang für ein kleines, handelsorientiertes Land wie die Schweiz oder Österreich kaum sinnvoll ist. Implementieren die Nachbarn Kapazitätsmechanismen, dürfte es aus Gründen der längerfristigen Versorgungssicherheit sinnvoll sein, ebenfalls einen solchen Mechanismus einzuführen. Doch selbst bei der Gestaltung und Umsetzung des Mechanismus ist eine Koordination mit den Nachbarn sinnvoll. Mindestens theoretisch weist das Modell der Kapazitätsverpflichtung gegenüber den Preis-basierten Mechanismen wesentliche Vorteile auf, da die induzierten Marktverzerrungen geringer sind: Erstens resultiert keine explizite (verzerrende) Preisbegrenzung auf dem Strommarkt, zweitens kann das Modell relativ einfach sämtliche Kraftwerkstypen und verbrauchsseitigen Massnahmen in wettbewerblicher Form integrieren und drittens tendiert der Preis am Kapazitätsmarkt gegen null, wenn auf absehbare Zeit ausreichend Kraftwerkskapazitäten zur Verfügung stehen (Meister 2013). Die Herausforderungen eines solchen Kapazitätsmarktes liegen in der Komplexität und dem Aufwand. Gerade in einem kleinen Land wäre die isolierte Einführung ineffizient - nicht nur wegen administrativer Kosten. Während der Stromgrosshandel aufgrund der relativ grossen Grenzkapazitäten im Netz wesentlich durch das Angebot im Ausland beeinflusst wird, weist ein rein inländischer Kapazitätsmarkt eine zu geringe Liquidität auf. Die Wahrscheinlichkeit ist hoch, dass inländische Anbieter die Preise auf dem Kapazitätsmarkt zu ihren Gunsten beeinflussen. Sinnvollerweise sollte der Kapazitätsmarkt eine Beschaffung im Ausland zulassen (natürlich unter Berücksichtigung allfälliger saisonabhängiger Netzengpässe).

\section{Schlussfolgerungen}

Kleine Strommärkte mit hohem Anteil Handel können ihr Marktdesign nicht autonom wählen. Gerade Länder wie die Schweiz oder Österreich mit einer faktischen Fremdbestimmung der Strompreise im Grosshandel sollten bei der Einführung von Kapazitätsmechanismen keinen Alleingang vornehmen. Die unilaterale Einführung eines Kapazitätsmechanismus wäre in jedem Fall ineffizient: Die inländischen Verbraucher finanzieren die Kosten des Mechanismus, erhalten hingegen keinen Gegenwert in Form tieferer Grosshandelspreise bzw. der Verhinderung von Preisspitzen (Knappheitspreisen). Umgekehrt dürfte es schwierig sein, bei einer Einführung von Kapazitätsmärkten in den Nachbarländern ab- 
seits zu stehen. Zwar können inländische Verbraucher von tieferen Preisen profitieren, doch erodieren die Investitionsanreize in inländische Kraftwerke. Die Einführung eines eigenen Kapazitätsmechanismus wird nötig, um langfristige Versorgungssicherheit zu schaffen. Auch bei der Umsetzung eines Mechanismus ist eine Koordination mit den Nachbarn sinnvoll. Denn ein auf das Inland beschränkter Kapazitätsmarkt wäre aufgrund der administrativen Aufwendungen, der fehlenden Liquidität und des mangelnden Wettbewerbs beim Kraftwerksangebot auf jeden Fall ineffizient.

\section{Literaturhinweise}

Barrera, F./Janssen, M./Riechmann, Ch. (2011): Kapazitätsmärkte: Aus der internationalen Praxis lernen?, in: Energiewirtschaftliche Tagesfragen, Jg. 61, Nr. 9, S. 8-12

BFE, Bundesamt für Energie (2013): Elektrizitätsstatistik, Bern.

Bothe, D./Richemann, Ch. (2008): Hohe Versorgungssicherheit bei Strom wertvoller Standortfaktor für Deutschland, in: Energiewirtschaftliche Tagesfragen, Jg. 58, Nr. 10.

Cramton, P./Stoft, S. (2008): Forward Reliability Markets: Less Risk, Less Market Power, More Efficiency, in: Utilities Policy, Jg. 16, S. 194-201.

Cramton, P./Stoft, S. (2006): The Convergence of Market Designs for Adequate Generating Capacity with Special Attention to the CAISO's Resource Adequacy Problem, A White Paper for the Electricity Oversight Board, Massachusetts Institute of Technology, California.

EWI, Energiewirtschaftliches Institut an der Universität zu Köln (2012): Untersuchungen zu einem zukunftsfähigen Strommarktdesign, Köln.

Frontier Economics (2008): Kosten von Stromversorgungsunterbrechungen, im Auftrag von RWE AG, Präsentation vom 4. Juli, London.

Paul, L./Tirole, J. (2007): Reliability and competitive electricity markets, in: RAND Journal of Economics, Jg. 38, Nr. 1, S. 60-84.

Meister, U. (2013): Keine Energiewende im Alleingang: Wie die Schweiz mit Ökostrom und Kapazitätsmärkten umgehen soll, Avenir Suisse, Zürich.

Pfeifenberger, J./Spees, K./Schumacher, A. (2009): A Comparison of PJM's RPM with Alternative Energy and Capacity Market Designs, The Brattle Group, Prepared for PJM Interconnection.

Siegmeier, J. (2011): Kapazitätsinstrumente in einem von erneuerbaren Energien geprägten Stromsystem, Dresden University of Technology, Chair of Energy Economics und Berlin University of Technology, Workgroup for Infrastructure Policy (WIP), Electricity Markets Working Papers, WP-EM-45.

Tietjen, O. (2012): Kapazitätsmärkte: Hintergründe und Varianten mit Fokus auf einen emissionsarmen deutschen Strommarkt, Studie, Germanwatch, Berlin.

Urs Meister, Dr. oec. publ., ist Projektleiter und Mitglied des Kaders des Think Tanks Avenir Suisse.

Anschrift: Avenir Suisse, Rotbuchstrasse 46, CH-8037 Zürich. Tel.: +41 (0) 44-445-90-09, Fax: +41 (0)44-445-90-01, E-Mail: urs.meister@avenir-suisse.ch 